NEWS

\title{
Plagiarism scandal grows in Iran
}

\section{Investigation finds more cases of duplication in publications co-authored by ministers and senior officials.}

\section{EXCLUSIVE}

Nature has uncovered further instances of apparent plagiarism in papers co-authored by government ministers and senior officials in Iran. The spate of new examples raises questions about whether such incidents are symptomatic of conditions also common in other developing countries - such as difficulties with English or pressure to acquire academic credentials as a prerequisite for promotion - or whether they are also linked specifically to the Iranian regime, where growth of a merit-based university culture has been undermined by political appointments and purges of reformminded scientists (see page 699).

An earlier probe ${ }^{1,2}$ revealed extensive plagiarism in a paper co-authored by transport minster Hamid Behbahani and four papers coauthored by science minister Kamran Daneshjou. The revelations received wide coverage in the Iranian media and blogosphere. Scientists inside and outside the country have called for investigations, as well as for stronger ethical oversight in Iran's research institutions.

Daneshjou, a mechanical engineer at the Iran University of Science \& Technology (IUST) in Tehran, was head of the interior-ministry office that oversaw this year's disputed election that kept President Mahmoud Ahmadinejad in power. In October, the Iranian parliament's commission for science and education held an informal inquiry into the four Daneshjou papers. Although it made no official conclusion, it effectively cleared Daneshjou after his co-author, IUST colleague Majid Shahravi, took responsibility for the papers' contents in the Iranian media - although both Shahravi and some members of the commission also maintain that the papers contained originality. Three of the four papers have now been retracted by the journals in question - the fourth was in an Iranian journal.

The paper ${ }^{3}$ by Behbahani, an IUST researcher who supervised Ahmadinejad's $\mathrm{PhD}$, has not been investigated, although it seems to be almost entirely put together from three earlier articles by different authors ${ }^{2}$. It was retracted by the journal Transport in October.

Behbahani has publicly said that the paper did not constitute plagiarism because only parts of the article were identical to earlier work. He challenged the allegations of plagiarism, calling them a "media attack, far from fairness and integrity" and "an illegitimate accusation".

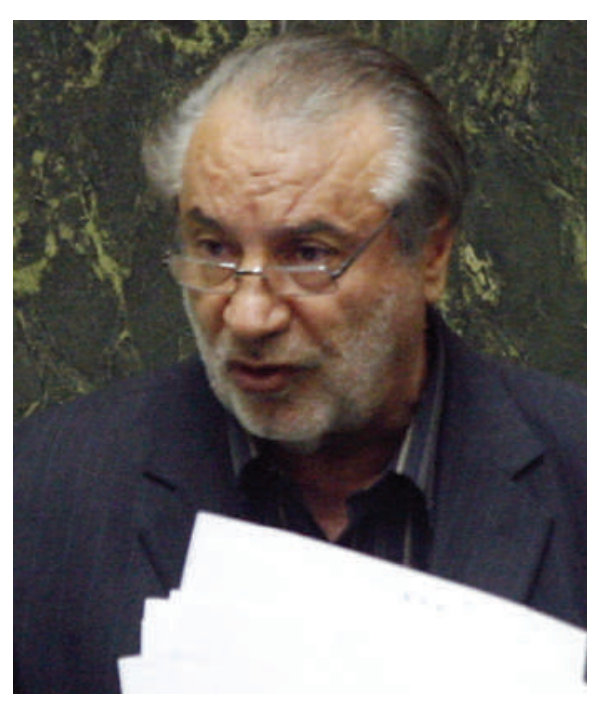

Research papers co-authored by Hamid

Behbahani contain text from other works.

Nature has now uncovered yet more instances of apparent plagiarism in papers from Behbahani and some of his co-authors.

One paper $^{4}$ on asphalt-road resistance - by Behbahani's Transport co-authors Hassan Ziari, a deputy minister of roads and transportation whom Daneshjou recently appointed as head of Payame Noor University in Tehran, and Mohammed Khabiri, then a PhD student at the IUST - contains many sections that are identical to a 2005 paper $^{5}$ by scientists in Pakistan.

And two 2008 papers $^{6,7}$ on strengthening asphalt roads, co-authored by Behbahani and Ziari with PhD student Shams Noubakhat, also contain duplicated material. The first ${ }^{6}$ includes multiple passages from three earlier papers ${ }^{8-10}$ and the second ${ }^{7}$ is also largely taken from three other papers ${ }^{10-12}$.

One scientist familiar with the field, who asked to remain anonymous, says that he has difficulty making sense of the first paper's results, and that some data in $i^{6}$ are identical to those in one of the earlier papers by different researchers 8 . "That the two sets of results could be identical is improbable," he says. Behbahani and Ziari did not respond to requests for comment. Muhammad Atif Ramay, managing editor of the Journal of Applied Sciences Research in which both papers were published, says that the journal has withdrawn the articles from its website pending further investigation.

Also in question is a 2008 paper on modelling pollution in $\operatorname{Iran}^{13}$, which is co-authored by one of the 37 members of the Iranian Supreme Council of Cultural Revolution, Mohammad Ali Kaynejad, an environmental engineer at Sahand University of Technology in Tabriz, Iran. The paper almost entirely duplicates a 2001 conference paper ${ }^{14}$ on modelling pollution in Hungary.

The Iranian paper acknowledges the original source of the model, although the authors wrote that it was "tested via the simulation of a photochemical oxidant episode that took place in Tabriz, Iran in 2007". But Alison Tomlin, an environmental modeller at the University of Leeds, UK, and a co-author on the Hungary model, says that the Iranian paper contains "no new results" and "is definitely a copy". It includes computer simulations purportedly of Iranian data, but they match the Hungary figures - and the background map outlines Hungary, not Iran.

The first author of the Iranian paper, Esmaeil Fatehifar, an environmental engineer at Sahand University of Technology, places the responsibility on another member of the team. "He said these are measured data about Tabriz Petrochemical Complex," he says. "I thought he was right and accepted it." Fatehifar says he intends to cancel the team member's PhD plans. $\mathrm{He}$ adds that Kaynejad had "not seen that paper" even though his name is on it. Kaynejad did not respond to Nature's interview requests.

Questions have also been raised over work co-authored by Ali Reza Ali-Ahmadi, education minister in the previous government of Ahmadinejad. A 2006 paper ${ }^{15}$ on supply networks co-authored by him includes many sentences and paragraphs that are identical to those in three earlier papers ${ }^{16-18}$. Mika Ojala at Tampere University of Technology in Finland, a co-author on one of the earlier studies, says that in his opinion this is not coincidence. AliAhmadi could not be reached for comment. Babak Amiri, an IUST researcher and a coauthor on the paper, says that a draft version of the paper was accidentally submitted before it was checked by himself or Ali-Ahmadi. "I apologize for this big mistake," he says.

Nature has also learned that the US National Academy of Sciences earlier this year removed a chapter from a 2003 book $^{19}$ on a US-Iranian workshop. Ironically, the chapter, authored by Hassan Zohoor, secretary of the Academy of Sciences of the Islamic Republic of Iran, was 
called 'The impact of moral values on the promotion of science'. It was withdrawn because it substantially duplicated a 1999 paper $^{20}$ by Douglas Allchin, a historian and philosopher now at the University of Minnesota in Minneapolis.

Zohoor says that he never saw Allchin's paper, and that he only prepared a draft of the paper, leaving others in his office to "develop it and add the literature review". Zohoor says that the explanation of the staff member involved that the copying happened "quite accidentally and as a mere negligence" - is inadequate, and that he intends to write to Allchin to apologize. "In my entire life I've never copied anyone else's work," says Zohoor.

Declan Butler
Butler, D. Nature doi:10.1038/news.2009.945 (2009).

Butler, D. Nature 461, 578-579 (2009).

3. Ziari, H., Behbahani, H. \& Khabiri, M. M. Transport XXI, 207-212 (2006).

4. Ziari, H. \& Khabiri, M. M. J. Eng. Appl. Sci. 2, 33-37 (2007).

5. Kamal, M. A. Shazib, F. \& Yasin, B. J. East. Asia Soc. Transport. Stud. 6, 1329-1343 (2005).

6. Behbahani, H., Ziari, H. \& Noubakhat, S. J. Appl. Sci. Res. 4, 96-102 (2008).

7. Behbahani, H., Ziari, H. \& Noubakhat, S. J. Appl. Sci. Res. 4, 282-286 (2008).

8. Awwad, M. T. \& Shbeeb, L. Am. J. Appl. Sci. 4, 390-396 (2007).

9. Lucena, M. C. C., Soares, S. A. \& Soares, J. B. Mater. Res. 7, 529-534 (2004)

10.Emery, S. J. \& O'Connell, J. in Proc. 7th Conf. Asphalt Pavements for Southern Africa 29 August-2 September 1999, Victoria Falls, Zimbabwe (CAPSA, 1999).

11. Hofsink, W., Kong Kam Wa, N. Y. \& Dickinson, M. N. in Proc 8th Conf. on Asphalt Pavements for Southern Africa 12-16 September 2004, Sun City, South Africa (CAPSA, 2004).
COPENHAGEN LIVE

Nature's reporters blog from the United Nations climate conference.

go.nature.com/OEsfAa

\section{Exoplanet claim bites the dust}

Strike one planet from the list of 400-odd found around stars in other solar systems: a proposed planet near a star some 6 parsecs from Earth may not exist after all.

The finding is also a strike against a planet-seeking strategy called astrometry, which measures the side-to-side motion of a star on the sky to see whether any unseen bodies might be orbiting it. Ground-based astrometry has been used for more than a century, but none of the extrasolar planets it has detected has been verified in subsequent studies.

In May, Steven Pravdo of the Jet Propulsion Laboratory in Pasadena, California, and his colleagues raised fresh hopes for the technique when they announced an exoplanet, six times more massive than Jupiter, orbiting VB10, a star about one-thirteenth the mass of the Sun, using a telescope at the Palomar Observatory in southern California (S. Pravdo and S. Shaklan Astrophys. J. $700,623-632 ; 2009)$. But now a group led by Jacob Bean at the Georg-August University in Gottingen, Germany, has used a different approach, and found nothing. "The planet is not there," says Bean.

Bean and his colleagues used a well-honed technique called radial velocity, which has found most of the extrasolar planets detected so far. The method looks for shifts in the lines of a star's absorption spectrum to track its motion towards and away from Earth, which would be caused by the influence of a planet.

Radial-velocity measurements typically exploit the visible bands of the electromagnetic spectrum. But VB10 is a very dim star and gives off most of its light as infrared radiation. At the Very
Large Telescope in Chile, Bean placed a gas cell filled with ammonia in the path of the starlight, enabling him to calibrate the instrument for the infrared.

"We would definitely have seen a significant amount of variation in our data if [the planet] was there," says Bean, who has submitted the work to the Astrophysical Journal (J. L. Bean et al. Astrophys. J. preprint at http://fr.arxiv.org/abs/0912.0003; 2009).

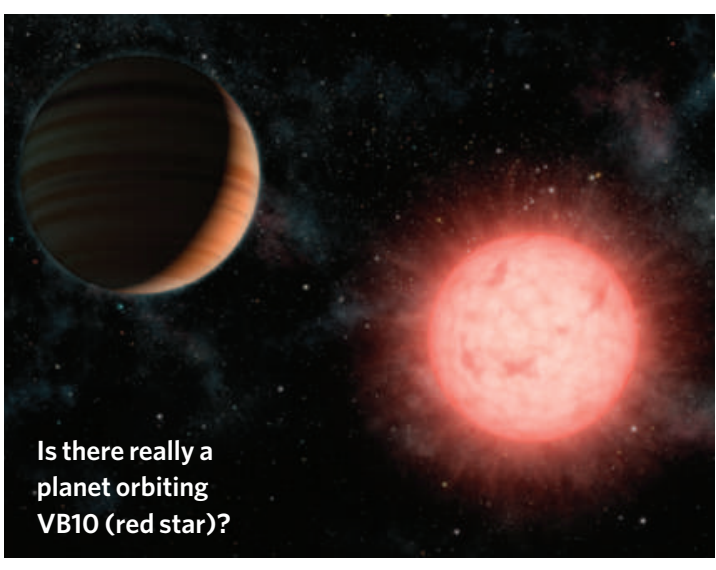

Pravdo says that Bean and his colleagues "may be correct, but there is hyperbole in their rejection of our candidate planet". Bean's paper, for instance, only rules out the presence of any planet that is at least three times more massive than Jupiter, says Pravdo, adding that the work "limits certain orbits for possible planets but not all planets".

"Unfortunately, astrometry is a very difficult business," counters Bean, explaining that Earth's atmosphere can introduce distortions that affect the measurements. Astrometrists rely on watching a field of stars about the same
12. Hanyu, A., Ueno, S., Kasahara, A. \& Saito, K. J. East. Asia Soc. Transport. Stud. 6, 1153-1167 (2005).

13. Fatehifar, E., Alizadeh Osalu, A., Kaynejad, M. A. \& Elkamel, A. in Proc. 3rd IASME/WSEAS Int. Conf. Energy \&Environment 23-25 February 2008, Univ. Cambridge, 330-335 (2008)

14. Lagzi, A. S. et al. in Air Pollution Modelling and Simulation (ed. Sportisse, B.) 264-273 (Springer, 2002).

15. Aliahmadi, A. R., Jafari, M. \& Amiri, B. in Proc. 2nd National Conf. Logistics \& Supply Chain 20-21 November 2006, Tehran (2006).

16. Hallikas, J., Karvonen, I., Pulkkinen, U., Virolainen, V.-M. \& Tuominen, M. Int. J. Prod. Econ. 90, 47-58 (2004).

17. Ojala, M. \& Hallikas, J. Int. J. Prod. Econ. 104, 201-213 (2006). 18. Harland, C., Brenchley, R. \& Walker, H. J. Purchasing Supply Management 9, 51-62 (2003)

19. The Experiences and Challenges of Science and Ethics: Proceedings of an American-Iranian Workshop (NAS, 2003). 20.Allchin, D. Sci. Educ. 8, 1-12 (1999).

See Editorial, page 699. distance away as the target star to calibrate their measurements, and that can be tricky, says Alessandro Sozzetti, an astrometry expert at the Turin Observatory in Italy. "Even if we think we have selected a good set of reference stars," he says, "we may still be limited by atmospheric effects that cause an extra jitter" in the motion of those stars.

Alan Boss, an exoplanet expert at the Carnegie Institution of Washington, agrees. He points to the well-known 'detection' of 1963, when Dutch astronomer Piet van de Kamp used astrometry to claim that two planets were orbiting Barnard's Star - a finding disproved a decade later. The dispute over the VB10 planet, says Boss, "is another example of how hard it is to detect extrasolar planets using astrometry from the ground".

Astronomers expect astrometry to work much better above the distorting effects of the atmosphere. Two space missions in the works - the European Space Agency's GAIA, due to launch in 2012, and NASA's Space Interferometry Mission, the launch date for which is yet to be set - will use the technique to search for planets as small as Earth around Sunlike stars, says Sozzetti. More significantly, astrometry can yield the mass of a planet, whereas radial velocity only puts a lower limit on it.

Bean admits that astronomers might one day find a planet around VB10 if they scrutinize the star long and hard enough. “The main lesson from VB10," says Boss, is that a lot of high-quality data are needed to be sure that an exoplanet is present. Katharine Sanderson 